\title{
EL ESTUDIO DE LA BIODIVERSIDAD EN SISTEMAS AGRÍCOLAS, UNA NECESIDAD URGENTE
}

\author{
The study of biodiversity in agricultural systems, an urgent necessity
}

Juan Fernando Escobar-Ibáñez ${ }^{1,2 *}$

${ }^{1}$ Estancia posdoctoral - Maestría en Ciencias en Biosistemática y Manejo de Recursos Naturales y Agrícolas, Centro Universitario de Ciencias Biológicas y Agropecuarias, Universidad de Guadalajara. Camino Ramón Padilla Sánchez No. 2100, predio Las Agujas, Zapopan, Jalisco 45200. ${ }^{2}$ GNÓSIS - Naturaleza con Ciencia A.C.

Autor para correspondencia: juanf.escobar@academicos.udg.mx

\section{Resumen}

Los sistemas agrícolas son uno de los usos de suelo más expandidos en el mundo y una de las principales amenazas para la biodiversidad, ya que son el principal factor de deforestación en sitios de gran diversidad biológica y vierten una gran cantidad de contaminantes al ambiente. Debido a su gran extensión y al inminente aumento de la misma, es fundamental evaluar la biodiversidad en sistemas agrícolas, ya que éstos son elementos dominantes en los paisajes actuales de gran parte del mundo. Por ello, el objetivo de este ensayo es introducir algunos aspectos básicos del estudio de la biodiversidad en sistemas agrícolas y señalar la necesidad urgente de desarrollar estudios de la biodiversidad asociada a los cultivos del estado de Jalisco. La información generada, junto con productores $y$ tomadores de decisiones, permitirá replantearnos el tipo de paisajes que deseamos en el futuro, estableciendo como prioridades las actividades productivas sostenibles, el mantenimiento de la vegetación natural para la conservación de la biodiversidad y de los servicios que ésta provee, así como del propio bienestar humano.
Palabras clave: Antropoceno, Cultivos, Diversidad, Paisajes modificados, Uso de suelo

\begin{abstract}
Agricultural systems are one of the most expanded land uses in the world and one of the main threats to biodiversity, since they are the main factor of deforestation in sites of great biological diversity and they pour a large amount of pollutants into the environment. Due to its great extension and its imminent increase, it is essential to assess biodiversity in agricultural systems, since these are dominant elements in the current landscapes of much of the world. For this reason, the objective of this essay is to introduce some basic aspects of the study of biodiversity in agricultural systems and to point out the urgent need to develop studies of the biodiversity associated with crops in the state of Jalisco. The information generated, together with producers and decision makers, will allow us to rethink the type of landscapes that we want in the future, establishing as priorities sustainable productive activities, the maintenance of natural
\end{abstract}


vegetation for the conservation of biodiversity and the services that it provides, as well as human well-being.

Key words: Anthropocene, Croplands, Diversity, Modified landscapes, Land use

Los sistemas agrícolas cubren alrededor del $12 \%$ de la superficie terrestre, siendo el uso de suelo de mayor superficie después de los pastos para ganadería $\quad(27 \%$ aproximadamente; Ramankutty et al. 2008). Debido a que son el principal factor de deforestación en los trópicos (DeFries et al. 2010), y uno de los principales responsables en la emisión de gases de efecto invernadero a la atmósfera y del vertimiento de herbicidas y agroquímicos al ambiente (Foley et al. 2011; Vermeulen et al. 2012), son considerados como una de las principales amenazas para la biodiversidad (Maxwell et al. 2016). En México, la tasa de deforestación es cinco veces mayor que el promedio mundial y se concentra en la región tropical del sur (Arroyo-Rodríguez et al. 2017), una de las de mayor biodiversidad en el país. Así que estamos frente a una realidad en la que los sistemas agrícolas y otras actividades humanas son un factor dominante en los paisajes actuales, lo que hace aún más indispensable y necesario su estudio (Melo et al. 2013).

Específicamente, el estado de Jalisco es el de mayor producción agrícola del país, con una superficie sembrada de aproximadamente 1'200,000 hectáreas. Por su extensión, los principales cultivos en el estado son: maíz (820,000 ha), caña de azúcar (85,739 ha), agave $(65,800$ ha) y sorgo para grano ( 31,240 ha) (SIAP 2017). Aunque es de reconocer el papel que el estado tiene en la producción de alimentos a nivel nacional, también es necesario señalar que dicha producción tiene numerosos impactos negativos en la biodiversidad que no han sido muy explorados. Por ello, el objetivo de este ensayo es introducir algunos aspectos básicos del estudio de la biodiversidad en sistemas agrícolas y señalar la necesidad urgente de desarrollar estudios de la biodiversidad asociada a los cultivos del estado de Jalisco.

A pesar de los impactos negativos que tienen los sistemas agrícolas en la biodiversidad, algunos cultivos ofrecen refugio para una gran cantidad de especies. $\mathrm{Su}$ capacidad de albergar biodiversidad depende de aspectos directamente relacionados con el cultivo y con las condiciones y el contexto espacial en donde éste se desarrolla (Bennett et al. 2006). Con respecto a las características del cultivo, algunos de los aspectos que son determinantes en la biodiversidad asociada son: la estructura del cultivo (herbáceo, arbustivo, arbóreo), la diversidad de especies cultivadas (monocultivo o policultivo), el manejo cultural (e.g., uso de agroquímicos), y el ciclo del cultivo (anual o perenne). Por ejemplo, en cultivos de nopal tunero de la región de los Llanos de Ojuelos en el estado de Jalisco, diferentes estudios han permitido reconocer que las huertas con menor manejo (e.g., reducción de la eliminación de vegetación asociada como hierbas, arbustos e incluso árboles, menor insumo de agroquímicos) sirven como refugio para una gran cantidad de especies de aves (muchas de ellas migratorias; Mellink et al. 2016) y de mamíferos, incluso para mamíferos microendémicos como el ratón de abazones rayado (Chaetodipus lineatus; Riojas-López et al. 2018). Sin embargo, para la mayoría de los cultivos en el estado no existe información acerca de 
estas variables y su relación con la biodiversidad, lo que limita nuestra comprensión y la capacidad de desarrollar propuestas de manejo en beneficio de la biodiversidad y del propio bienestar humano.

Entender las características de los cultivos que permiten la presencia de la diversidad es un paso importante. Sin embargo, es necesario considerar que los sistemas agrícolas no son sistemas aislados, sino que se encuentran interactuando con diferentes usos de suelo (e.g., habitacional, industrial, conservación) y diferentes elementos (e.g., cercas vivas, árboles aislados, caminos, remanentes de vegetación). Por lo anterior, para poder entender las dinámicas de la biodiversidad contenida en los paisajes agrícolas, es indispensable conocer el número de elementos y de usos de suelo presentes, así como la proporción que ocupa cada uno, lo que se conoce como composición paisajística. Además, también es necesario conocer el arreglo espacial que tienen éstos componentes, es decir, si se encuentran de manera continua o de manera fragmentada (Bennett et al. 2006). Cabe señalar que su importancia está en función del grupo de organismos que se estén evaluando y de su capacidad de dispersión en el paisaje, así como de sus requerimientos de hábitat y su sensibilidad a las perturbaciones (Fahrig et al. 2011). Una revisión reciente (Arroyo-Rodríguez et al. 2017) señala que los estudios a escala de paisaje en México están enfocados principalmente en plantas, mamíferos, y aves, así que existen muchos vacíos de información que atender.

Además de la importancia que tienen las prácticas agrícolas de cada cultivo y del paisaje en el que se encuentra inmerso para la biodiversidad, es importante señalar que la biodiversidad provee una gran cantidad de servicios ecosistémicos, cuya cantidad y calidad también están en función de las características del cultivo y del paisaje. Por lo anterior, es indispensable entender las variables dentro de los cultivos y las variables a nivel de paisaje que influyen en la biodiversidad, ya que los paisajes actuales están dominados por actividades humanas con algunos remanentes de vegetación y la expansión de las zonas agrícolas es inminente. Debido a que éstas variables cambian entre regiones y entre cultivos, la generación de información específica en el estado de Jalisco permitirá entender su relación con la biodiversidad y será el punto de partida para abrir el diálogo con agricultores y con tomadores de decisiones en la búsqueda de alternativas que permitan disminuir el impacto en la biodiversidad sin afectar la producción. Por ejemplo, en Asia se demostró que un manejo integral de plagas puede reducir el uso de pesticidas en un $50 \%$ (Pretty y Bharucha 2015). Por ello, para enfrentar la problemática actual de la producción alimentaria y la conservación de la biodiversidad, considero que el estudio de la biodiversidad en sistemas agrícolas es una necesidad urgente en el país y especialmente en el estado de Jalisco.

Las posibilidades y los enfoques para realizar estudios de la biodiversidad en sistemas agrícolas del estado de Jalisco son muy amplios y deben abordarse a la brevedad. Específicamente, considero necesario estudios que evalúen: (1) la biodiversidad en cultivos que no han sido estudiados en el estado (e.g., tejocote, guayaba) y en cultivos en continua expansión (e.g., caña de azúcar, aguacate, agave), (2) la biodiversidad en cultivos 
del mismo varietal bajo diferente manejo o en diferente ubicación geográfica, (3) la caracterización y cuantificación puntual de la intensificación agrícola y su impacto en la biodiversidad, (4) las implicaciones del cambio de tipo de cultivo (e.g., de maíz a caña de azúcar) o de componente (e.g., de remanente de vegetación a cultivo), y (5) la importancia de la composición y la heterogeneidad del paisaje en el movimiento y permanencia de las especies en paisajes dominados por cultivos poco estudiados. Esta información en conjunto con tomadores de decisiones y productores, permitirá que nos replanteemos el tipo de paisajes que deseamos en el futuro, estableciendo como prioridades las actividades productivas sostenibles, el mantenimiento de la vegetación natural para la conservación de la biodiversidad y de los servicios que ésta provee, así como el propio bienestar humano.

\section{Agradecimientos}

Este manuscrito forma parte de una estancia posdoctoral (CVU 416899), por lo que agradezco ampliamente al Consejo Nacional de Ciencia y Tecnología por la beca otorgada para el desarrollo de la misma. Así mismo, agradezco a la Dra. Mónica Riojas López por sus comentarios a una versión anterior y a la Dra. Natalia Mesa Sierra por sus comentarios para mejorar este manuscrito.

\section{Literatura citada}

Arroyo-Rodríguez, V., C.E. Moreno, y C. Galán-Acedo. (2017). La Ecología Del Paisaje En México: Logros, Desafíos y Oportunidades En Las Ciencias Biológicas.
Revista Mexicana de

Biodiversidad, 88, 42-51. https://doi.org/10.1016/j.rmb.2017. 10.004

Bennett, A.F., J.Q. Radford, y A. Haslem. (2006). Properties of Land Mosaics: Implications for Nature Conservation in Agricultural Environments. Biological Conservation, 133, 250-64. doi:10.1016/j.biocon.2006.06.008

DeFries, R.S., T. Rudel, M. Uriarte, y M. Hansen. (2010). Deforestation Driven by Urban Population Growth and Agricultural Trade in the Twenty-First Century. Nature Geoscience, 3, 178-81. https://doi.org/10.1038/ngeo756

Fahrig, L., J. Baudry, L. Brotons, F.G. Burel, T.O. Crist, R.J. Fuller, C. Sirami, G.M. Siriwardena, y J.L. Martin. (2011). Functional Landscape Heterogeneity and Animal Biodiversity in Agricultural Landscapes: Heterogeneity and Biodiversity. Ecology Letters, 14, 101-12. doi: 10.1111/j.1461-0248.2010.01559.x

Foley, J.A., N. Ramankutty, K.A. Brauman, E.S. Cassidy, J.S. Gerber, M. Johnston, N.D. Mueller, C. O'Connell, D.K. Ray, P.C. West, C. Balzer, E.M. Bennett, S.R. Carpenter, J. Hill, C. Monfreda, S. Polasky, J. Rockström, J. Sheehan, S. Siebert, D. Tilman, y D.P.M. Zaks. (2011). Solutions for a Cultivated Planet. Nature, 478, 337-42. doi:10.1038/nature10452

Maxwell, S.L., R.A. Fuller, T.M. Brooks, y J.E.M. Watson. (2016). The Ravages of Guns, Nets and Bulldozers. Nature, 143-45. http://dx.doi.org/10.1016/j.jaridenv .2015 .07 .0 
Mellink, E., M.E. Riojas-López, y P. Giraudoux. (2016). A Neglected Opportunity for Bird Conservation: The Value of a Perennial, Semiarid Agroecosystem in the Llanos de Ojuelos, Central Mexico. Journal of Arid Environments, 124, 1-9. $\mathrm{http}: / / \mathrm{dx}$.doi.org/10.1016/j.jaridenv .2015 .07 .005

Melo, F.P.L., V. Arroyo-Rodríguez, L. Fahrig, M. Martínez-Ramos, y M. Tabarelli. (2013. On the Hope for Biodiversity-Friendly Tropical Landscapes. Trends in Ecology \& Evolution, 28, 462-68. http://dx.doi.org/10.1016/j.tree.201 3.01 .001

Pretty J., y Z.P. Bharucha, (2015). Integrated Pest Management for Sustainable Intensification of Agriculture in Asia and Africa. Insects, 6, 152-182. doi:10.3390/insects6010152

Ramankutty, N., A.T. Evan, C. Monfreda, y J.A. Foley. (2008). Farming the Planet: 1. Geographic Distribution of Global Agricultural Lands in the Year 2000: GLOBAL AGRICULTURAL LANDS IN 2000. Global Biogeochemical Cycles, 11, 377-92. doi:10.1029/2007GB002952

Riojas-López, M.E., E. Mellink, y J. Luévano. (2018). A Semiarid Fruit Agroecosystem as a ConservationFriendly Option for Small Mammals in an Anthropized Landscape in Mexico. Ecological Applications, 28, 495-507. doi: 10.1002/eap. 1663

(SIAP) Servicio de Información Agroalimentaria y Pesquera. Estadística de La Producción Agrícola de 2017. Datos Abiertos,
Estadística de Producción Agrícola. Accesado el 24 de abril, 2019

(http://infosiap.siap.gob.mx/gobmx /datosAbiertos.php).

Vermeulen, S.J., B.M. Campbell, y J.S.I. Ingram. (2012). Climate Change and Food Systems. Annual Review of Environment and Resources, 37, 195-222. doi: 10.1146/annurev-environ-020411130608 